\title{
Costs and Treatment Patterns Among Patients with Atopic Dermatitis Using Advanced Therapies in the United States: Analysis of a Retrospective Claims Database
}

\author{
Lawrence F. Eichenfield - Marco DiBonaventura (D) - Jason Xenakis • \\ Marie-Helene Lafeuille · Mei Sheng Duh • Iman Fakih • \\ Mark Levenberg · Joseph C. Cappelleri · Vanja Sikirica
}

Received: April 24, 2020 / Published online: June 30, 2020

(C) The Author(s) 2020

\section{ABSTRACT}

Introduction: For many, atopic dermatitis (AD) is not adequately controlled with topical regimens. This analysis examined treatment using advanced therapies and associated costs.

Methods: The IQVIA Health Plan Claims data set was analyzed. Patients aged $\geq 12$ years with AD who newly initiated advanced therapy after the availability of dupilumab (March 28, 2017) and had $\geq 6$ months continuous enrollment

Digital Features To view digital features for this article go to https://doi.org/10.6084/m9.figshare.12489146.

L. F. Eichenfield

Department of Dermatology and Pediatrics, UC San Diego, San Diego, CA, USA

L. F. Eichenfield

Rady Children's Hospital, San Diego, San Diego, CA, USA

M. DiBonaventura $(\bowtie) \cdot J$. Xenakis

Pfizer Inc., New York, NY, USA

e-mail: marco.dibonaventura@pfizer.com

M.-H. Lafeuille · I. Fakih

Analysis Group, Montreal, QC, Canada

M. S. Duh

Analysis Group, Boston, MA, USA

M. Levenberg · V. Sikirica

Pfizer Inc., Collegeville, PA, USA

J. C. Cappelleri

Pfizer Inc., Groton, CT, USA before and after their first advanced therapy claim (index date) were included. Advanced therapies included dupilumab, systemic corticosteroids (SCSs), systemic immunosuppressants (SISs), and phototherapy. A multivariate regression model was used to predict annualized follow-up healthcare costs.

Results: In total, 1980 patients were included (61.1\% female; mean age, 41.2 years [SD, 17.4]; $11.3 \%<18$ years). Pre-index date, $65.2 \%$ of patients used topical corticosteroids (TCSs; $40.7 \%$ and $32.1 \%$ used medium and high potency, respectively). The most common advanced therapy was SCSs $(N=1453$ [73.4\%]; $69.2 \%$ prednisone) followed by dupilumab $(N=265$ [13.4\%]), SISs $(N=99$ [5.0\%]; 47.5\% methotrexate), and phototherapy $(N=163$ [8.2\%]). Of patients treated with dupilumab, SISs, and phototherapy, $17.4 \%, 26.3 \%$, and $14.1 \%$, respectively, were prescribed SCSs postindex date. Overall, $62.6 \%$ of patients initiating SCSs, $49.1 \%$ initiating dupilumab, 64.6\% initiating SISs, and $36.2 \%$ initiating phototherapy were prescribed TCSs post-index date. Mean annualized total costs (SD) post-index date were $\$ 20,722(\$ 47,014)$ : $\$ 11,196(\$ 41,549)$ in medical costs $(\$ 7973[\$ 35,133]$ in outpatient visit costs) and $\$ 9526(\$ 21,612)$ in pharmacy costs. Mean annualized total cost (SD) varied significantly $(P<0.05)$ by index treatment: dupilumab, \$36,505 (\$14,028); SCSs, \$17,924 $(\$ 49,019) ;$ SISs, $\$ 24,762(\$ 47,583)$; phototherapy, and $\$ 17,549(\$ 57,238)$. 
Conclusions: Switching to combination therapy with SCSs and TCSs was common within 6 months of initiating advanced therapy in patients with AD. Patients also incurred significant pharmacy and outpatient costs. These results highlight the difficulty of managing $\mathrm{AD}$ with these existing treatment options.

Keywords: Atopic dermatitis; Corticosteroids; Costs; Dupilumab; Immunosuppressants; Phototherapy

\section{Key Summary Points}

Why carry out this study?

Many patients with atopic dermatitis cannot manage their disease with topical treatments, requiring advanced therapy.

This analysis examined advanced therapy (systemic corticosteroids, systemic immunosuppressants, phototherapy, and dupilumab) use between March 28, 2017, and July 31,2018 , and associated costs in patients with atopic dermatitis aged $\geq 12$ years using the IQVIA Health Plan Claims data set.

\section{What was learned from the study?}

Patients initiating an advanced treatment often switch to or add on another advanced treatment and often use topical treatments concomitantly.

Adherence rates to advanced treatments are not optimal.

The patterns of use of advanced therapies represent a significant burden to the healthcare system, with costs representing approximately $\$ 20,000$ per patient per year.

\section{INTRODUCTION}

Atopic dermatitis (AD) is a chronic, relapsing inflammatory skin condition characterized by dry, pruritic skin and eczematous lesions [1]. The disease is relatively common, affecting approximately $20 \%$ of children and $10 \%$ of adults in the USA $[2,3]$. AD is associated with several comorbidities, such as anxiety and depression, and it has a profound impact on patients' health-related quality of life $[4,5]$.

Many of those with AD can achieve adequate disease control with some combination of nonpharmacologic topical interventions (e.g., emollients) and/or prescription topical therapies such as corticosteroids, calcineurin inhibitors, and phosphodiesterase 4 inhibitors $[6,7]$. For patients whose $\mathrm{AD}$ cannot be controlled with these therapies, treatment intensification with phototherapy or systemic immunosuppressants (SISs) can be considered (subsequently referred to as "advanced therapies") [8]. SISs, which include methotrexate, cyclosporine, azathioprine, and mycophenolate mofetil, are recommended as potential treatment options even though they are not approved by the US Food and Drug Administration (FDA) for AD and have long-term toxicity issues [8]. Dupilumab, an interleukin (IL)-4 receptor antagonist that blocks IL-4 and IL-13, is a subcutaneously administered biologic therapy that represents another advanced therapy option for patients with moderate-to-severe $\mathrm{AD}$ after its recent approval by the US FDA in 2017 [9, 10].

As the management of AD has evolved since the introduction of dupilumab, the primary goal of this study was to evaluate the current real-world treatment patterns for patients with moderate-to-severe $\mathrm{AD}$, including the distribution of advanced treatments used, adherence to and persistence with those treatments, and the frequency of combination therapy in a cohort of US commercially insured patients with AD. Recent studies showed that annual costs for patients with $\mathrm{AD}$ in commercial health plans who were treated with either phototherapy or systemic treatments (i.e., patients with "more severe $\mathrm{AD}^{\prime \prime}$ ) were approximately $\$ 15,000$ $[11,12]$. However, these studies were conducted 
using data periods prior to the availability of dupilumab. The current study will be used to provide updated information on the cost burden of $\mathrm{AD}$ and provide current cost figures after the introduction of dupilumab, including stratification by the specific advanced treatment(s) used. Collectively, these data will provide a current view on the management and costs associated with moderate-to-severe AD.

\section{METHODS}

\section{Data Source}

A retrospective cohort study was conducted using the IQVIA Health Plan Claims (formerly PharMetrics Plus) database. The IQVIA Health Plan Claims database is the largest non-payer owned integrated claims database of commercial insurers and consists of fully adjudicated medical (inpatient and outpatient diagnoses and procedures) and pharmacy (retail and mailorder prescriptions) claims for more than 105 million patients across the USA. It is generally representative of the commercially insured US population with respect to age and sex. Data are deidentified and compliant with the Health Insurance Portability and Accountability Act (HIPAA). This study was an analysis of secondary data and was exempt from institutional review board approval.

\section{Sample}

Patients with AD (ICD-9/ICD-10-CM: 691.8/ L20.x) who newly initiated a treatment associated with moderate-to-severe $\mathrm{AD}$ between March 28, 2017 (the date of approval of dupilumab), and July 31, 2018, were included. This period was selected to reflect the current treatment options because the introduction of dupilumab might have significantly changed the treatment paradigm and costs for patients with moderate-to-severe AD. Given the lack of clinical data in claims databases, the treatments received were used as a surrogate for disease severity, as done in prior research $[11,12]$. The first claim for a moderate-to-severe $\mathrm{AD}$ treatment (i.e., one of the following advanced therapies: phototherapy, dupilumab, systemic corticosteroid [SCS], or SIS [methotrexate, cyclosporine, azathioprine, or mycophenolate mofetil]) was considered the index date. A postindex date follow-up period of at least 6 months was used to assess the research questions (i.e., the 6 months after initiation of an advanced therapy). The specific inclusion criteria were as follows: (1) at least two AD diagnoses at any time during data availability (January 1, 2013-July 31, 2018), including one during the 6-month pre-index date baseline period (i.e., the 6 months before initiation of an advanced therapy); (2) at least 6 months of continuous eligibility before and after the index date; (3) at least 12 years of age on the index date; and (4) at least two claims for any treatment associated with moderate-to-severe AD on March 28, 2017, or later, with no claim for those specific agents in the baseline period. There were no exclusion criteria beyond not meeting these inclusion criteria.

\section{Measures}

\section{Treatment Cohort}

Patients were categorized into a treatment cohort based on the advanced treatment used on the index date: dupilumab, SCS, SIS, or phototherapy.

\section{Demographics and Health History}

Demographic information regarding the patients' age, sex, region, payer type, and insurance type on the index date was collected and reported. Comorbidities were evaluated during the 6-month baseline period and presented using the Quan-Charlson comorbidity index (Quan-CCI; score of 1-year mortality prediction; 0-24 scale with higher score indicating higher mortality) $[13,14]$.

\section{Treatment Patterns}

Persistence to the index treatment was calculated based on the time spent on therapy without a gap of $>60$ days (such a gap was used to define a discontinuation). Adherence was calculated using a proportion of days covered 
(PDC) method $[15,16]$. To identify the use of combination therapy, all AD-related treatments (both topical and systemic) were reported before and after the index treatment.

\section{Healthcare Resource Utilization and Costs}

Costs associated with outpatient visits, inpatient visits, emergency room visits, and other medical visits (including durable medical equipment use, dental care, and vision care) were recorded and annualized to represent allcause medical costs. Similarly, drug costs were reported and annualized as part of the all-cause pharmacy costs. Annualized costs were obtained by calculating monthly costs and then multiplying them by 12 . Total costs represent the sum of these costs.

\section{Statistical Analyses}

Descriptive analyses (frequencies and percentages for categorical variables; means, standard deviations [SD], and medians for continuous variables) were reported for demographics, health history, and treatment pattern analyses. Statistical differences ( $P$ values) in baseline characteristics and adherence measures across treatment cohorts were calculated using analysis of variance models [17] for continuous variables and chi-square tests [18] or Fisher exact tests [19] (if an expected count was less than 5) for categorical variables. Patient persistence with the index treatment was described using Kaplan-Meier rates and compared using logrank tests [20]. A multivariable linear regression model [21] based on the index treatment cohort and baseline variables was also used to predict annualized total follow-up health care costs. Cost outcomes were compared using adjusted mean cost differences and 95\% CIs estimated with gamma regression models, and $P$ values were reported. All analyses were conducted using SAS Enterprise Guide, version 7.15.

\section{RESULTS}

\section{Sample Characteristics}

A total of 1980 patients were newly initiated a treatment associated with moderate-to-severe $\mathrm{AD}$ (Fig. 1). The mean age (SD) of this sample was 41.2 years $(17.4 ; 88.7 \%$ were aged $\geq 18$ years $)$, $61.1 \%$ were female, and patients were disproportionately located in the South $(42.5 \%)$ versus the West $(12.2 \%)$ of the US. The distribution of patients in the index treatment cohorts was as follows: $73.4 \%$ were in the SCS cohort $(69.2 \%$ of whom initiated prednisone), $13.4 \%$ were in the dupilumab cohort, $8.2 \%$ were in the phototherapy cohort, and $5.0 \%$ were in the SIS cohort $(47.5 \%$ and $30.3 \%$ of whom initiated methotrexate and cyclosporine, respectively).

A few significant demographic differences were observed across treatment cohorts (Tables 1 and 2). Those initiating dupilumab were the least likely to be aged $<18$ years $(2.3 \%)$ and the most likely to have a preferred provider organization (PPO) insurance type $(89.8 \%)$ relative to other cohorts (both $P<0.05)$. Patients initiating phototherapy were least likely to be in the South (16.0\%) and most

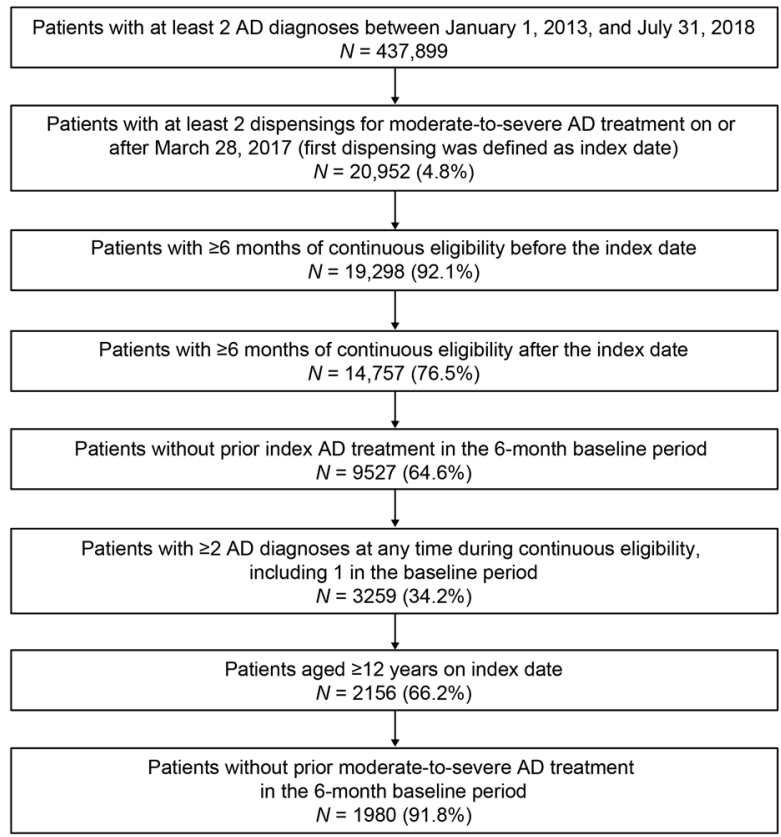

Fig. 1 Sample selection. $A D$ atopic dermatitis 


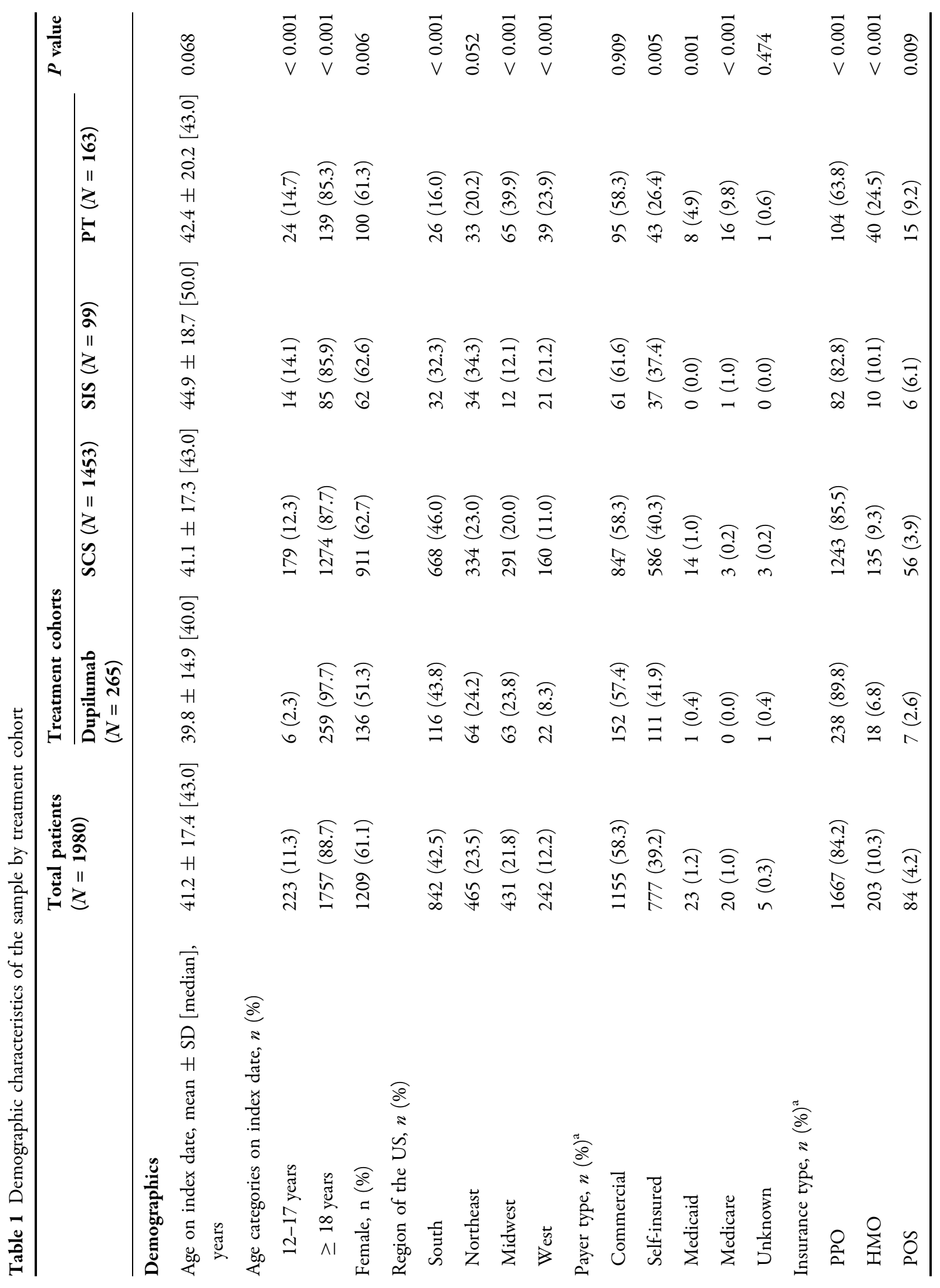




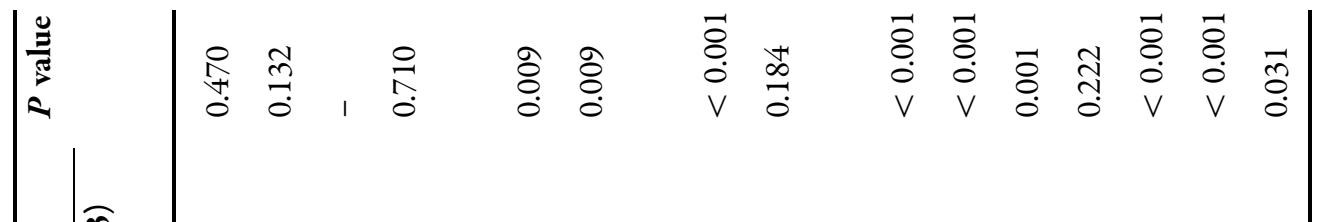

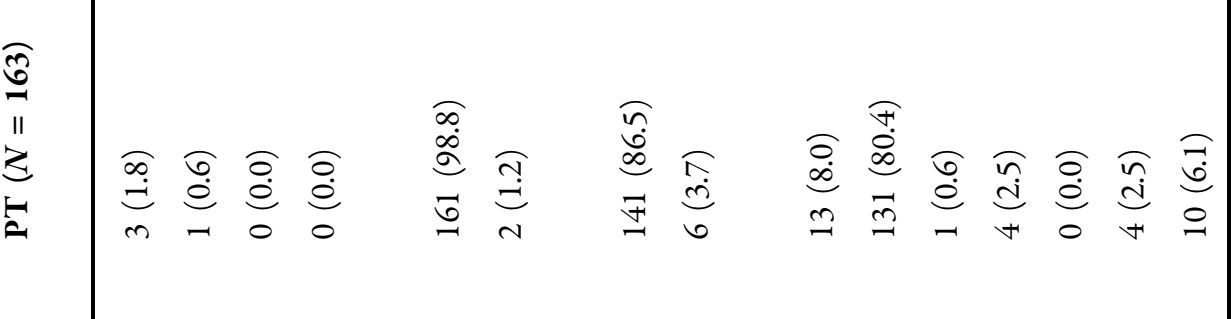

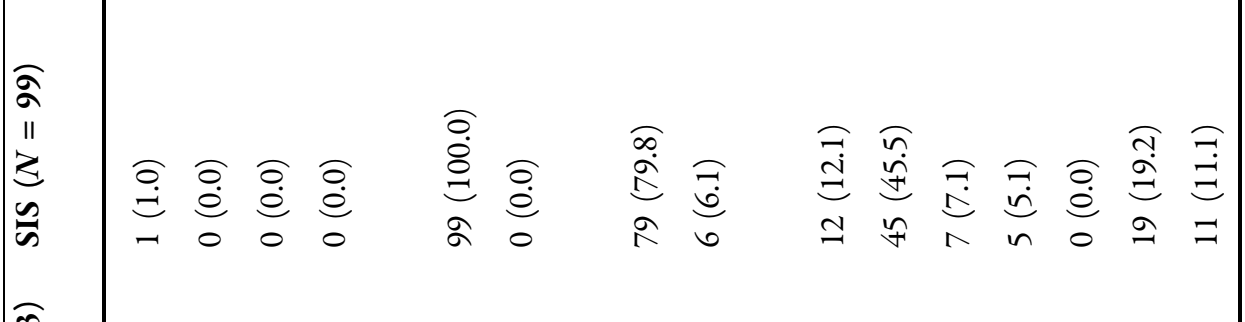

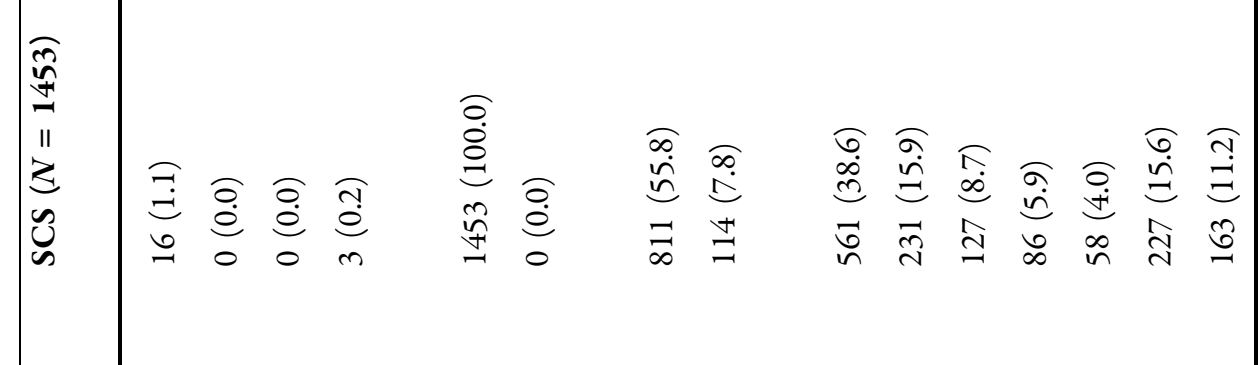

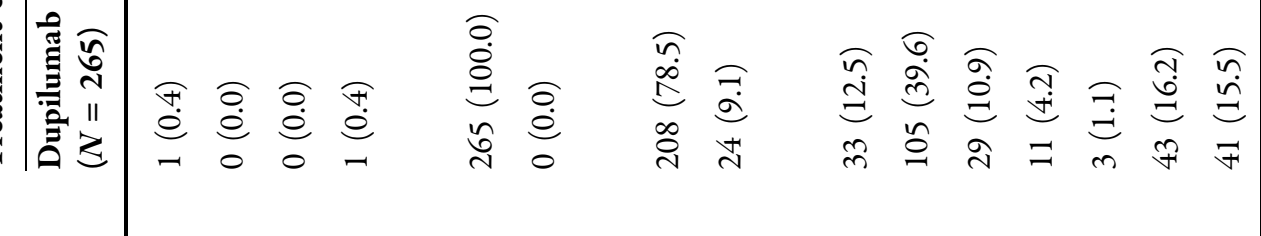

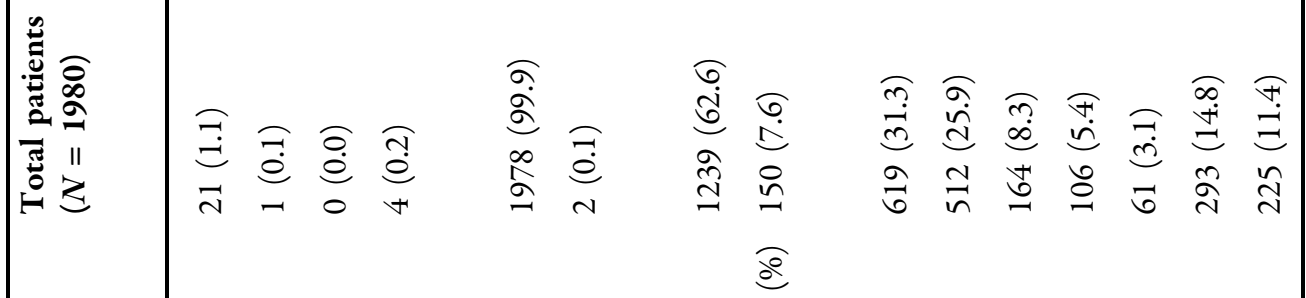

$$
\begin{aligned}
& \text { 尊 }
\end{aligned}
$$

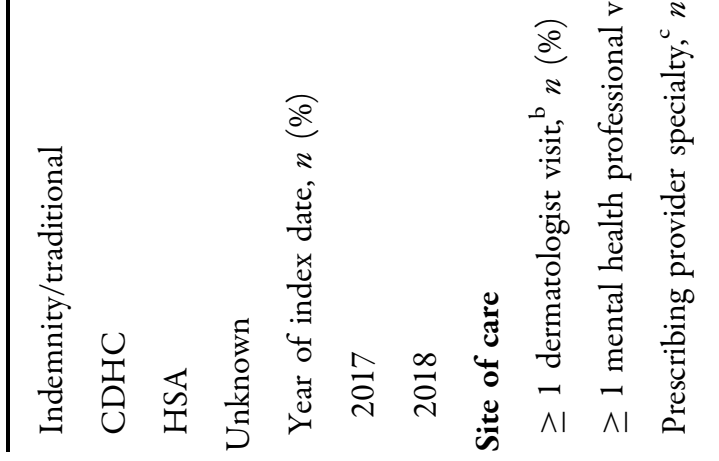

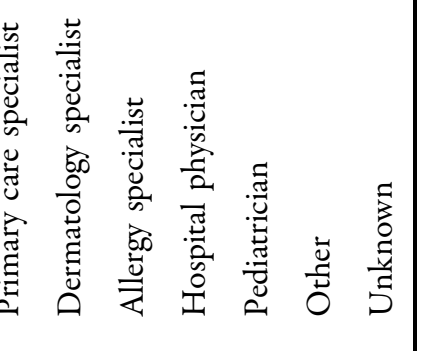




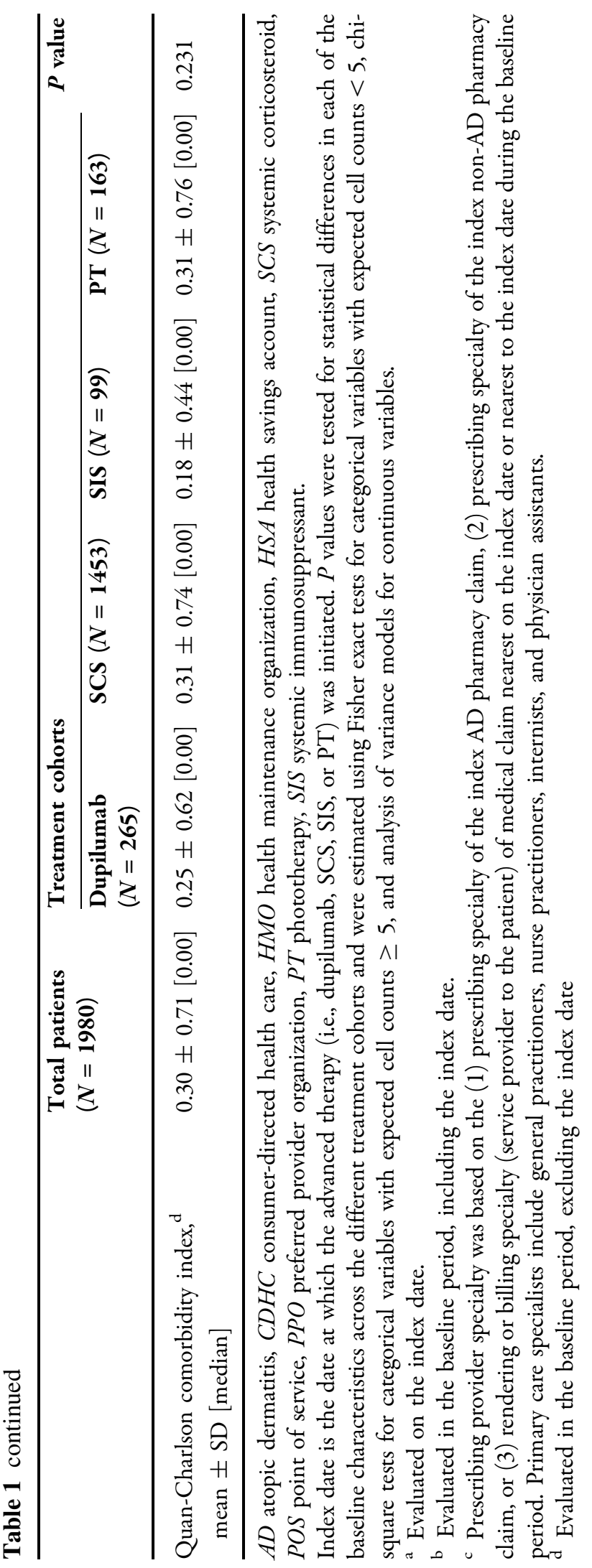


Table 2 Treatment history by treatment cohort

\begin{tabular}{|c|c|c|c|c|c|c|}
\hline & \multirow{2}{*}{$\begin{array}{l}\text { Total patients } \\
(N=1980)\end{array}$} & \multicolumn{4}{|c|}{ Treatment cohorts } & \multirow[t]{2}{*}{$P$ value } \\
\hline & & $\begin{array}{l}\text { Dupilumab } \\
(N=265)\end{array}$ & $\begin{array}{l}\text { SCS } \\
(N=1453)\end{array}$ & $\begin{array}{l}\text { SIS } \\
(N=99)\end{array}$ & $\begin{array}{l}\text { PT } \\
(N=163)\end{array}$ & \\
\hline \multicolumn{7}{|c|}{$\begin{array}{l}\text { Treatments initiated on the index } \\
\text { date, } n(\%)\end{array}$} \\
\hline SCS (total) & $1453(73.4)$ & $0(0.0)$ & $1453(100.0)$ & $0(0.0)$ & $0(0.0)$ & - \\
\hline Prednisone & $1006(50.8)$ & $0(0.0)$ & $1006(69.2)$ & $0(0.0)$ & $0(0.0)$ & - \\
\hline Methylprednisolone & $387(19.5)$ & $0(0.0)$ & $387(26.6)$ & $0(0.0)$ & $0(0.0)$ & - \\
\hline Dexamethasone & $30(1.5)$ & $0(0.0)$ & $30(2.1)$ & $0(0.0)$ & $0(0.0)$ & - \\
\hline Prednisolone & $16(0.8)$ & $0(0.0)$ & $16(1.1)$ & $0(0.0)$ & $0(0.0)$ & - \\
\hline Other & $14(0.7)$ & $0(0.0)$ & $14(1.0)$ & $0(0.0)$ & $0(0.0)$ & - \\
\hline SIS (total) & $99(5.0)$ & $0(0.0)$ & $0(0.0)$ & $99(100.0)$ & $0(0.0)$ & - \\
\hline Methotrexate & $47(2.4)$ & $0(0.0)$ & $0(0.0)$ & $47(47.5)$ & $0(0.0)$ & - \\
\hline Cyclosporine & $30(1.5)$ & $0(0.0)$ & $0(0.0)$ & $30(30.3)$ & $0(0.0)$ & - \\
\hline Mycophenolate mofetil & $16(0.8)$ & $0(0.0)$ & $0(0.0)$ & $16(16.2)$ & $0(0.0)$ & - \\
\hline Azathioprine & $6(0.3)$ & $0(0.0)$ & $0(0.0)$ & $6(6.1)$ & $0(0.0)$ & - \\
\hline Dupilumab & $265(13.4)$ & $265(100.0)$ & $0(0.0)$ & $0(0.0)$ & $0(0.0)$ & - \\
\hline Phototherapy & $163(8.2)$ & $0(0.0)$ & $0(0.0)$ & $0(0.0)$ & $163(100.0)$ & - \\
\hline \multicolumn{7}{|c|}{$\begin{array}{l}\text { Treatments used in the } 6 \text { months } \\
\text { before the index date, } n(\%)\end{array}$} \\
\hline TCS & $1290(65.2)$ & $186(70.2)$ & $962(66.2)$ & $69(69.7)$ & $73(44.8)$ & $<0.001$ \\
\hline Low potency & $243(12.3)$ & $33(12.5)$ & $180(12.4)$ & $11(11.1)$ & $19(11.7)$ & 0.976 \\
\hline Medium potency & $806(40.7)$ & $127(47.9)$ & $584(40.2)$ & $43(43.4)$ & $52(31.9)$ & 0.010 \\
\hline High potency & $635(32.1)$ & $105(39.6)$ & $448(30.8)$ & $41(41.4)$ & $41(25.2)$ & 0.001 \\
\hline Unknown potency & $24(1.2)$ & $5(1.9)$ & $15(1.0)$ & $1(1.0)$ & $3(1.8)$ & 0.437 \\
\hline Tacrolimus ointment & $112(5.7)$ & $32(12.1)$ & $62(4.3)$ & $7(7.1)$ & $11(6.7)$ & $<0.001$ \\
\hline Pimecrolimus cream & $79(4.0)$ & $17(6.4)$ & $54(3.7)$ & $3(3.0)$ & $5(3.1)$ & 0.215 \\
\hline Crisaborole ointment & $62(3.1)$ & $21(7.9)$ & $35(2.4)$ & $5(5.1)$ & $1(0.6)$ & $<0.001$ \\
\hline
\end{tabular}

PT phototherapy, SCS systemic corticosteroid, SIS systemic immunosuppressant, TCS topical corticosteroid.

Index date is the date at which the advanced therapy (i.e., dupilumab, SCS, SIS, or PT) was initiated. $P$ values tested for statistical differences in each of the treatment history variable across the different treatment cohorts and were estimated using Fisher's exact tests for categorical variables with expected cell counts $<5$ and chi-square tests for categorical variables with expected cell counts $\geq 5$

likely to be in the Midwest (39.9\%), in the West (23.9\%), on Medicare $(9.8 \%)$, or have a health maintenance organization insurance type
(24.5\%) relative to other subgroups (all $P<0.05)$. Compared with the SCS and phototherapy cohorts, the dupilumab and SIS 
cohorts were more likely to have used topical corticosteroids (TCSs; particularly high-potency TCSs, $39.6 \%$ and $41.4 \%$, respectively), tacrolimus $(12.1 \%$ and $7.1 \%)$, and crisaborole $(7.9 \%$ and $5.1 \%$ ) prior to their current therapy (all $P<0.05$; Table 2).

\section{Treatment Patterns}

It was not uncommon for patients to use a subsequent advanced treatment after the index date (either in combination with their index treatment or as a switch from their index treatment). Forty-four (44.4\%) and 54 (20.4\%) patients in the SIS and dupilumab cohorts used an additional treatment, respectively, with SCSs ( $n=23,52.3 \%$ and $n=46,85.2 \%$, respectively) being the most frequent (Table 3). A total of 220 $(15.1 \%)$ and $29(17.8 \%)$ patients in the SCS and phototherapy cohorts used an additional advanced treatment after the index date, respectively. For the SCS cohort, this was most frequently dupilumab $(n=114 ; 51.8 \%$ of those who used an additional treatment); for the phototherapy cohort, this was most frequently SCSs $(n=21 ; 72.4 \%)$.

Significant differences in persistence with oral or injectable index treatment associated with moderate-to-severe $\mathrm{AD}$ were observed among treatments at 3, 6, 9, and 12 months $(P<0.05 ; \quad$ Fig. 2$)$. By 6 months, $21.9 \%$ of patients initiating dupilumab, 63.6\% initiating SISs, and $97.7 \%$ initiating SCSs discontinued therapy, defined by a gap in treatment $>60$ days. By 12 months, these figures were $25.0 \%, 72.2 \%$, and $98.4 \%$ (Fig. 2). The median time to discontinuation was 12 days for SCSs and 124 days for SISs. Adherence to the index treatment during the first 6 months, based on mean PDC values, was 0.81, 0.58, and 0.13 for dupilumab, SISs, and SCSs, respectively (Fig. 3a). Hence, $68.7 \%, 32.3 \%$, and $1.1 \%$ of patients treated with dupilumab, SISs, and SCSs, respectively, were classified as "adherent" based on the standard of PDC $\geq 0.80$ (Fig. 3b). Significant differences $(P<0.05)$ were observed among index treatments for the proportion of patients considered adherent and the mean PDC at 6 months.

\section{Healthcare Costs}

Mean per-patient annualized all-cause healthcare costs were $\$ 20,722$ (SD, $\$ 47,014$; median, $\$ 7470$ ) across all patients with moderate-tosevere $\mathrm{AD}$ during the follow-up period. These costs were relatively evenly split between medical costs $(\$ 11,196)$ and pharmacy costs (\$9526). However, there was significant $(P<0.05$ for all-cause medical costs and allcause pharmacy costs) variability in cost depending on the treatment cohort (Fig. 4), with total costs ranging from $\$ 36,505$ for the dupilumab cohort to $\$ 17,549$ for the phototherapy cohort. Total mean annualized costs for the dupilumab cohort were primarily a function of pharmacy costs $(\$ 32,885)$; medical costs for this cohort (\$3620) were notably lower than those of other cohorts $(P<0.05)$, which varied between $\$ 12,066$ and $\$ 14,944$.

Additional post hoc analyses were undertaken to predict follow-up costs (Fig. 5). The strongest predictors of higher annualized follow-up costs were treatment cohort (dupilumab, specifically, contributed to an increase of $\$ 19,435 ; P<0.05)$, baseline Quan-CCI scores $(\$ 15,495$ for each increase of 1 point in the score; $P<0.05)$, and baseline all-cause total costs ( $\$ 653$ for each increase of $\$ 1000$ in baseline costs; $P<0.05)$. Conversely, the strongest predictors of lower annualized follow-up costs were the presence of an atopic march condition (allergic rhinitis, asthma, food allergies) $(-\$ 8457 ; P<0.05)$ and the number of inpatient visits per year during baseline $(-\$ 13,723$; $P<0.05)$.

\section{DISCUSSION}

The aim of this study was to provide a more current assessment of the treatment patterns and costs associated with managing moderateto-severe AD in the United States. SCSs were the most common advanced treatment initiated (prednisone, specifically) followed by dupilumab, phototherapy, and SISs (methotrexate and cyclosporine, specifically), although many of these patients concomitantly used topical therapies. Treatment selection may be partially a 
Table 3 Treatments used in the follow-up period by treatment cohort

\begin{tabular}{|c|c|c|c|c|}
\hline \multirow{2}{*}{ Treatment patterns } & \multicolumn{4}{|c|}{ Treatment cohort } \\
\hline & $\begin{array}{l}\text { Dupilumab } \\
(N=265)\end{array}$ & $\begin{array}{l}\text { SCS } \\
(N=1453)\end{array}$ & $\begin{array}{l}\text { SIS } \\
(N=99)\end{array}$ & $\begin{array}{l}\text { PT } \\
(N=163)\end{array}$ \\
\hline $\begin{array}{l}\text { Next treatment received during the follow-up } \\
\text { period, } n(\%)^{a}\end{array}$ & $54(20.4)$ & $220(15.1)$ & $44(44.4)$ & $29(17.8)$ \\
\hline Dupilumab & - & $114(7.8)$ & $18(18.2)$ & $4(2.5)$ \\
\hline Systemic corticosteroids (total) & $46(17.4)$ & - & $23(23.2)$ & $21(12.9)$ \\
\hline Prednisone & $27(10.2)$ & - & $20(20.2)$ & $16(9.8)$ \\
\hline Methylprednisolone & $15(5.7)$ & - & $2(2.0)$ & $4(2.5)$ \\
\hline Dexamethasone & $2(0.8)$ & - & $1(1.0)$ & $0(0.0)$ \\
\hline Prednisolone & $0(0.0)$ & - & $0(0.0)$ & $1(0.6)$ \\
\hline Cyclosporine & $4(1.5)$ & $14(1.0)$ & - & $1(0.6)$ \\
\hline Mycophenolate mofetil & $1(0.4)$ & $9(0.6)$ & - & $0(0.0)$ \\
\hline Azathioprine & $2(0.8)$ & $6(0.4)$ & - & $0(0.0)$ \\
\hline Methotrexate & $0(0.0)$ & $48(3.3)$ & - & $3(1.8)$ \\
\hline Phototherapy & $1(0.4)$ & $29(2.0)$ & $3(3.0)$ & - \\
\hline \multicolumn{5}{|l|}{ All treatments during follow-upn $n(\%)^{b}$} \\
\hline \multicolumn{5}{|l|}{ Treatments for moderate-to-severe $\mathrm{AD}$} \\
\hline Dupilumab & $265(100.0)$ & $124(8.5)$ & $21(21.2)$ & $5(3.1)$ \\
\hline Systemic corticosteroids & $46(17.4)$ & $1453(100.0)$ & $26(26.3)$ & $23(14.1)$ \\
\hline Prednisone & $33(12.5)$ & $1227(84.4)$ & $23(23.2)$ & $21(12.9)$ \\
\hline Methylprednisolone & $16(6.0)$ & $602(41.4)$ & $2(2.0)$ & $4(2.5)$ \\
\hline Dexamethasone & $2(0.8)$ & $69(4.7)$ & $1(1.0)$ & $0(0.0)$ \\
\hline Prednisolone & $0(0.0)$ & $25(1.7)$ & $0(0.0)$ & $1(0.6)$ \\
\hline Other & $2(0.8)$ & $25(1.7)$ & $0(0.0)$ & $0(0.0)$ \\
\hline Cyclosporine & $4(1.5)$ & $18(1.2)$ & $30(30.3)$ & $2(1.2)$ \\
\hline Mycophenolate mofetil & $2(0.8)$ & $10(0.7)$ & $19(19.2)$ & $0(0.0)$ \\
\hline Azathioprine & $2(0.8)$ & $9(0.6)$ & $6(6.1)$ & $0(0.0)$ \\
\hline Methotrexate & $0(0.0)$ & $50(3.4)$ & $48(48.5)$ & $5(3.1)$ \\
\hline Phototherapy & $1(0.4)$ & $31(2.1)$ & $3(3.0)$ & $163(100.0)$ \\
\hline \multicolumn{5}{|l|}{ Topical treatments } \\
\hline TCS & $130(49.1)$ & $910(62.6)$ & $64(64.6)$ & $59(36.2)$ \\
\hline Low potency & $26(9.8)$ & $206(14.2)$ & $10(10.1)$ & $12(7.4)$ \\
\hline Medium potency & $79(29.8)$ & $588(40.5)$ & $41(41.4)$ & $40(24.5)$ \\
\hline
\end{tabular}


Table 3 continued

\begin{tabular}{lllll}
\hline Treatment patterns & Treatment cohort & & & \\
\cline { 2 - 5 } & $\begin{array}{l}\text { Dupilumab } \\
(\boldsymbol{N}=\mathbf{2 6 5})\end{array}$ & $\begin{array}{l}\text { SCS } \\
(\boldsymbol{N}=\mathbf{1 4 5 3})\end{array}$ & $\begin{array}{l}\text { SIS } \\
(\boldsymbol{N}=\mathbf{9 9})\end{array}$ & $\begin{array}{l}\text { PT } \\
(\boldsymbol{N}=\mathbf{1 6 3})\end{array}$ \\
\hline High potency & $60(22.6)$ & $499(34.3)$ & $41(41.4)$ & $32(19.6)$ \\
Unknown potency & $2(0.8)$ & $27(1.9)$ & $0(0.0)$ & $2(1.2)$ \\
Tacrolimus ointment & $30(11.3)$ & $87(6.0)$ & $17(17.2)$ & $10(6.1)$ \\
Crisaborole ointment & $19(7.2)$ & $66(4.5)$ & $8(8.1)$ & $5(3.1)$ \\
Pimecrolimus cream & $13(4.9)$ & $60(4.1)$ & $5(5.1)$ & $5(3.1)$ \\
\hline
\end{tabular}

$A D$ atopic dermatitis; PT phototherapy; SCS systemic corticosteroid; SIS systemic immunosuppressant; TCS topical corticosteroid

a "Next treatment received" is the subsequent advanced therapy after the original therapy was initiated.

b "All treatments during the follow-up" refers to all subsequent therapies, regardless of the order and whether they were advanced

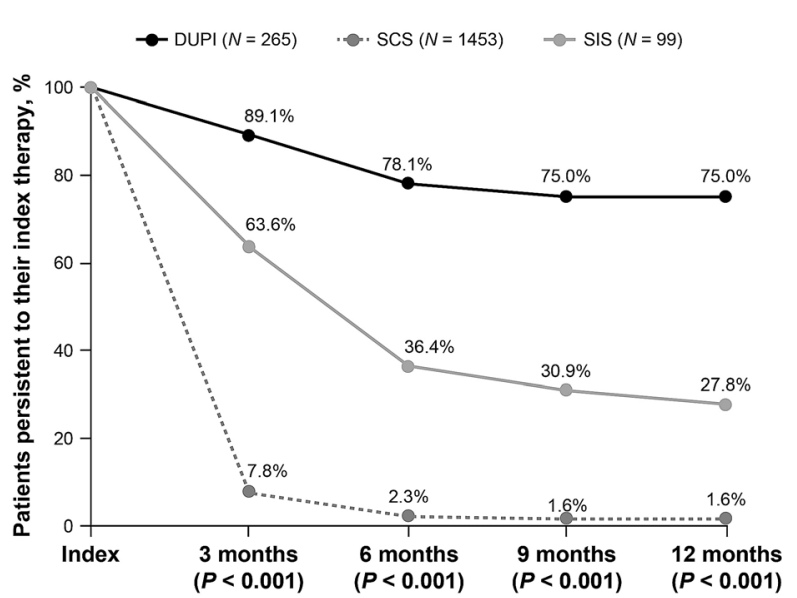

Fig. 2 Kaplan-Meier rates of persistence by treatment cohort. DUPI dupilumab; SCS systemic corticosteroid; SIS systemic immunosuppressant. Persistence was defined as the absence of a 60-day treatment gap. Phototherapy was excluded from this analysis because it is neither an oral nor an injectable treatment

function of access. For example, dupilumab was not indicated for adolescents during the current study period, which would likely explain the notably lower use among those aged $<18$ years [9]. Phototherapy requires access to healthcare professionals with specific medical equipment,

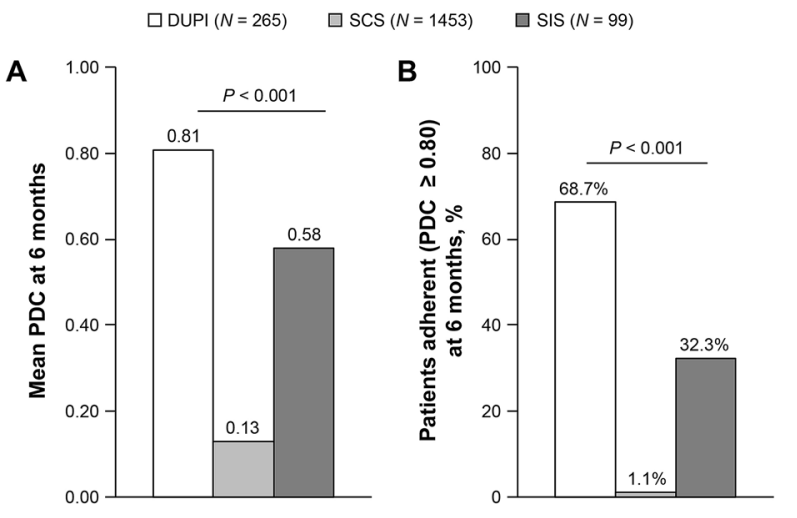

Fig. 3 Adherence to index treatment for moderate-tosevere $\mathrm{AD}$ during the first 6 months of follow-up: a based on mean PDC, $\mathbf{b}$ based on percentage of patients with PDC $\geq 0.80$. $A D$ atopic dermatitis, DUPI dupilumab, $S C S$ systemic corticosteroid, $P D C$ proportion of days covered, $S I S$ systemic immunosuppressant. $P$ values tested for statistical differences using chi-square tests for proportion of patients with $\mathrm{PDC} \geq 0.80$ and analysis of variance models for mean PDC. Phototherapy was excluded from this analysis because it is neither an oral nor an injectable treatment

which may be more difficult in certain areas of the country. In general, the distribution of advanced treatments is difficult to put into 


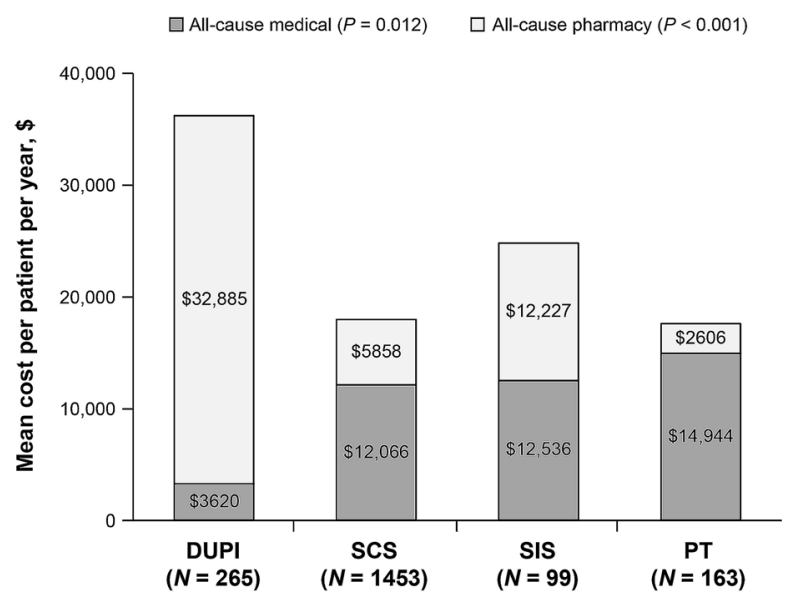

Fig. 4 Mean annualized all-cause health care costs by treatment cohort for patients initiating treatment for moderate-to-severe AD. DUPI dupilumab; SCS systemic corticosteroid; SIS systemic immunosuppressants; PT phototherapy. $P$ values tested for statistical differences in costs across the different treatment cohorts and were estimated using analysis of variance models

context because the current study is, to our knowledge, the first to examine treatment patterns after the approval of dupilumab.

However, most patients were treated with SCSs, despite American Academy of Dermatology (AAD) guideline recommendations to avoid them as a treatment option because of their unfavorable benefit-risk profile in $\mathrm{AD}$ [8]. The use of SCSs in the treatment of patients with $\mathrm{AD}$ could be related to SCSs's rapid clearing of AD [22]. The quick adoption of dupilumab, as it was the second most prescribed advanced treatment so soon after its approval, potentially illustrates the historical difficulty in treating these patients with the predupilumab armamentarium.

The switching and persistence data also indicate the challenges in treating patients who have moderate-to-severe AD. Approximately $20-45 \%$ of patients who newly initiated dupilumab or SISs used an additional advanced treatment after the index date, with SCSs being the most common option. Persistence rates were highest for dupilumab, although $22 \%$ of patients still discontinued treatment within 6 months. Understanding the reasons for discontinuation (e.g., safety events, loss of response) was beyond the scope of the study and the abilities of a commercial insurance data set; however, patients in the SISs treatment cohort were more likely to discontinue within 6 months. In part, this may be due to AAD guideline recommendations to discontinue systemic immunosuppressant treatment (e.g., cyclosporine, azathioprine) after skin clearance is achieved to avoid long-term treatment risks [8]. The low persistence rates of SCSs suggest mostly short-term use of these treatments, indicative of concerns regarding toxicity associated with long-term use and consistent with AAD guidelines [8]. However, there was a small proportion of patients that remained on SCS treatment without any substantial gap, potentially exposing them to adverse events resulting from long-term use [8].

Findings were similar for adherence. Adherence rates were highest for the dupilumab cohort (however, only $68.7 \%$ of patients were classified as adherent, defined as PDC $\geq 0.8$ during 6 months of follow-up) with notably lower adherence for SISs (only 32.3\% of patients were classified as adherent). This could have been related to the long-term toxic effects of SIS use and the reticence of patients to take them as frequently as prescribed. Unsurprisingly, given the short-term use of SCSs, adherence rates were incredibly low. Taken together, these results suggest that adherence may be an issue with patients who have $\mathrm{AD}$ who are using advanced therapies.

The current study also provided an update with respect to the costs of $\mathrm{AD}$ since the introduction of dupilumab. Substantial costs, estimated to be $>\$ 20,000$ per patient with AD per year in our analysis, suggest that costs have increased since the publication of results of prior studies [11, 12]. However, to our knowledge, this is the first time that costs have been compared as a function of the index treatment. Patients in the dupilumab cohort had the highest total costs, but this was disproportionately because of pharmacy costs; medical costs were notably lower than for other treatment cohorts, although they only partially offset the high pharmacy costs. No traditional clinical efficacy measures were available in the data set, but it is possible that dupilumab's clinical efficacy reduced medical costs (e.g., 


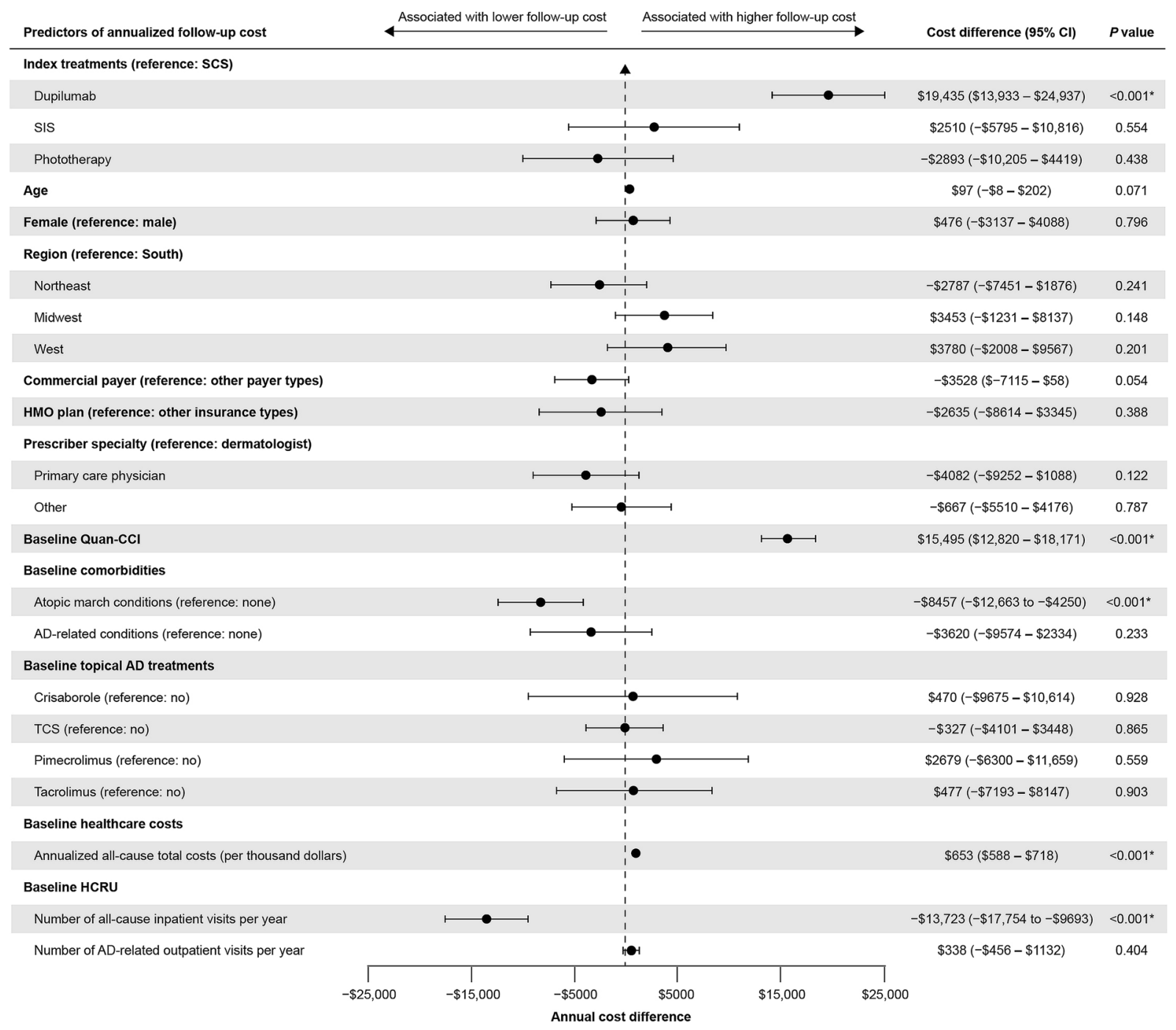

Fig. 5 Predictors of annualized all-cause health care costs in the follow-up period. $A D$ atopic dermatitis; $H C R U$ healthcare resource utilization; $H M O$ health maintenance

hospitalizations, outpatient visits) more than other treatments did, although total costs remained higher for dupilumab than for other treatment cohorts. Published results of metaanalyses have suggested greater clinical efficacy of dupilumab compared with cyclosporine, which may have translated to lower real-world medical costs [23]. More research is necessary to understand and compare real-world effectiveness among treatment options. organization; SCS systemic corticosteroid; SIS systemic immunosuppressants; Quan-CCI Quan-Charlson Comorbidity Index; TCS topical corticosteroid. ${ }^{*} P<0.05$

\section{Limitations}

This study has several limitations. Classification of moderate-to-severe $\mathrm{AD}$ was based entirely on the class of medication or treatment used because of the lack of disease severity data available, such as the Eczema Area and Severity Index or percentage of body surface area affected. This could have resulted in the inclusion of patients with mild disease who had a flare treated with SCSs or in the exclusion of patients with severe disease who, for whatever reason, 
had not yet progressed to an advanced therapy regimen per the treating clinician's discretion. Similarly, caution should be exercised when examining differences across treatment cohorts because there might have been unobserved selection bias that would not have been possible to control (e.g., patients with dupilumab as their index treatment may have had more severe disease than patients with phototherapy as their index treatment). The healthcare costs available from the IQVIA Health Plan Claims database were limited to those for outpatient visits, inpatient visits, emergency room visits, and other medical visits and did not include indirect costs. Furthermore, the current study was designed to examine overall costs over time, with the analysis stratified according to the different treatment cohorts; it was not designed to compare directly between treatment cohorts. Future studies should aim to examine these direct comparisons and include indirect healthcare costs. The current study focused on a postdupilumab period to determine current treatment patterns. However, the period immediately after the approval of dupilumab may not be fully representative of the current postdupilumab environment because it is possible that some patients may not have had access to dupilumab immediately when it became available.

\section{CONCLUSIONS}

The results of the current study highlight some of the challenges of treating patients with moderate-to-severe AD. Patients initiating an advanced treatment often switched to or addedon an advanced treatment after the index date. Topical therapies are also routinely used concomitantly with these advanced treatments. Almost two-thirds of patients who initiated SISs and a quarter of those who initiated dupilumab discontinued within 6 months. Even for those who remained, adherence rates are not optimal. These patients with $\mathrm{AD}$ also represent a significant burden to the healthcare system, with costs representing approximately $\$ 20,000$ per patient per year. Additional treatment options could help improve disease management and reduce costs for these patients.

\section{ACKNOWLEDGEMENTS}

Funding. This study and the Rapid Service Fee were funded by Pfizer Inc.

Medical Writing, Editorial, and Other Assistance. Medical writing support under the guidance of the authors was provided by Juan Sanchez-Cortes, $\mathrm{PhD}$, at ApotheCom, San Francisco, CA, and was funded by Pfizer Inc., New York, NY, in accordance with Good Publication Practice (GPP3) guidelines (Ann Intern Med. 2015;163:461-464).

Authorship. All named authors meet the International Committee of Medical Journal Editors (ICMJE) criteria for authorship for this article, take responsibility for the integrity of the work as a whole, and have given their approval for this version to be published.

Disclosures. Lawrence F. Eichenfield has served as a scientific adviser and/or clinical study investigator for Pfizer Inc., AbbVie, Almirall, Amgen, Asana, Dermavant, Dermira, DS Biopharma, Eli Lilly, Forte, Galderma, Glenmark, Incyte, LEO Pharma, Matrisys, Novartis, Ortho Dermatologics, Regeneron, Sanofi Genzyme, and UCB. Marie-Helene Lafeuille, Mei Sheng Duh, and Iman Fakih are employees of Analysis Group, which received research funding from Pfizer Inc. Marco DiBonaventura, Jason Xenakis, Mark Levenberg, Joseph C. Cappelleri, and Vanja Sikirica are employees and stockholders of Pfizer Inc.

Compliance with Ethics Guidelines. Data are identified and compliant with the Health Insurance Portability and Accountability Act (HIPAA). This study was an analysis of secondary data and was exempt from institutional review board approval.

Data Availability. The datasets generated during and/or analyzed during the current 
study are not publicly available due to a data use agreement between IQVIA and the authors but are available from IQVIA on reasonable request.

Open Access. This article is licensed under a Creative Commons Attribution-NonCommercial 4.0 International License, which permits any non-commercial use, sharing, adaptation, distribution and reproduction in any medium or format, as long as you give appropriate credit to the original author(s) and the source, provide a link to the Creative Commons licence, and indicate if changes were made. The images or other third party material in this article are included in the article's Creative Commons licence, unless indicated otherwise in a credit line to the material. If material is not included in the article's Creative Commons licence and your intended use is not permitted by statutory regulation or exceeds the permitted use, you will need to obtain permission directly from the copyright holder. To view a copy of this licence, visit http:// creativecommons.org/licenses/by-nc/4.0/.

\section{REFERENCES}

1. Boguniewicz M, Fonacier L, Guttman-Yassky E, Ong PY, Silverberg J, Farrar JR. Atopic dermatitis yardstick: practical recommendations for an evolving therapeutic landscape. Ann Allergy Asthma Immunol. 2018;120(10-22):e2.

2. Odhiambo JA, Williams HC, Clayton TO, Robertson CF, Asher MI, ISAAC Phase Three Study Group. Global variations in prevalence of eczema symptoms in children from ISAAC Phase Three. J Allergy Clin Immunol. 2009;124:1251-8.e23.

3. Silverberg JI, Hanifin JM. Adult eczema prevalence and associations with asthma and other health and demographic factors: a US population-based study. J Allergy Clin Immunol. 2013;132:1132-8.

4. Ronnstad ATM, Halling-Overgaard AS, Hamann CR, Skov L, Egeberg A, Thyssen JP. Association of atopic dermatitis with depression, anxiety, and suicidal ideation in children and adults: a systematic review and meta-analysis. J Am Acad Dermatol. 2018;79(448-56):e30.

5. Drucker AM, Wang AR, Li WQ, Sevetson E, Block JK, Qureshi AA. The burden of atopic dermatitis: summary of a report for the National Eczema Association. J Invest Dermatol. 2017;137:26-30.

6. Eichenfield LF, Tom WL, Berger TG, et al. Guidelines of care for the management of atopic dermatitis: section 2. Management and treatment of atopic dermatitis with topical therapies. J Am Acad Dermatol. 2014;71:116-32.

7. Johnson BB, Franco AI, Beck LA, Prezzano JC. Treatment-resistant atopic dermatitis: challenges and solutions. Clin Cosmet Investig Dermatol. 2019;12:181-92.

8. Sidbury R, Davis DM, Cohen DE, American Academy of Dermatology, et al. Guidelines of care for the management of atopic dermatitis: section 3 . Management and treatment with phototherapy and systemic agents. J Am Acad Dermatol. 2014;71: 327-49.

9. FDA approves new eczema drug Dupixent [press release]. Silver Spring, MD: US Food and Drug Administration. 2017.

10. Gooderham MJ, Hong HC, Eshtiaghi P, Papp KA. Dupilumab: a review of its use in the treatment of atopic dermatitis. J Am Acad Dermatol. 2018;78: S28.

11. Shrestha S, Miao R, Wang L, Chao J, Yuce H, Wei W. Burden of atopic dermatitis in the United States: analysis of healthcare claims data in the commercial, Medicare, and Medi-Cal databases. Adv Ther. 2017;34:1989-2006.

12. Drucker AM, Qureshi AA, Amand C, et al. Health care resource utilization and costs among adults with atopic dermatitis in the United States: a claims-based analysis. J Allergy Clin Immunol Pract. 2018;6:1342-8.

13. Quan H, Li B, Couris CM, et al. Updating and validating the Charlson comorbidity index and score for risk adjustment in hospital discharge abstracts using data from 6 countries. Am J Epidemiol. 2011;173:676-82.

14. Quan H, Sundararajan V, Halfon P, et al. Coding algorithms for defining comorbidities in ICD-9-CM and ICD-10 administrative data. Med Care. 2005;43:1130-9.

15. Osterberg L, Blaschke T. Adherence to medication. N Engl J Med. 2005;353:487-97.

16. Karve S, Cleves MA, Helm M, Hudson TJ, West DS, Martin BC. Prospective validation of eight different adherence measures for use with administrative claims data among patients with schizophrenia. Value Health. 2009;12:989-95. 
17. Stohle L, Wold S. Analysis of variance (ANOVA). Chemometr Intell Lab Syst. 1989;6:259-72.

18. Cochran WG. The Chi square test of goodness of fit. Ann Math Stat. 1952;23:315-45.

19. Agresti A. A survey of exact inference for contingency tables. Stat Sci. 1992;7:131-53.

20. Kleinbaum DG, Klein M. Kaplan-Meier survival curves and the log-rank test. In: Analysis Survival, A Self-Learning Text. 3rd ed. New York: Springer Science +Business Media; 2012. p. 55-96.
21. Gelman A, Hill J. Data Analysis Using Regression and Multilevel/Hierarchical Models. New York: Cambridge University Press; 2006.

22. Arkwright PD, Motala C, Subramanian H, et al. Management of difficult-to-treat atopic dermatitis. J Allergy Clin Immunol Pract. 2013;1:142-51.

23. Ariëns LFM, Gadkari A, van Os-Medendorp H, et al. Dupilumab versus cyclosporine for the treatment of moderate-to-severe atopic dermatitis in adults: indirect comparison using the eczema area and severity index. Acta Derm Venereol. 2019;99:851-7. 